\title{
Non-invasive recording of mitral valve flow velocity patterns using pulsed Doppler echocardiography Application to diagnosis and evaluation of mitral valve disease
}

\author{
D. KALMANSON, COLETTE VEYRAT, F. BOUCHAREINE, \\ AND A. DEGROOTE
}

From the Department of Cardiology, Fondation Ophtalmologique A. de Rothschild, Paris, France

A new technique using pulsed Doppler echocardiography for non-invasive recording of blood flow velocity patterns at the site of the mitral annulus is described. In a control group of 30 subjects in whom an organic or functional lesion of the mitral valve had been ruled out, the normal flow velocity pattern was found to be similar to that obtained either experimentally by other authors using an implanted electromagnetic cuff flowmeter or clinically by our group using the transseptal catheterisation continuous wave Doppler technique. In 31 patients with confirmed mitral valve disease, the mitral valve flow velocity patterns were correlated with the clinical and haemodynamic data, and in 10 cases the patterns were compared with the flow velocity curves recorded in the same patients using the Doppler ultrasonic transseptal catheterisation of the mitral valve. In most cases, characteristic anomalies of these patterns were noted and could be related to the type of the lesion, stenosis, regurgitation, or a combination of these.

Specific patterns of mitral valve flow velocity profiles were shown to correlate satisfactorily with the degree of the lesion, with some reservations for mitral regurgitation. Though not fully quantitative, the pulsed Doppler technique is a new and promising non-invasive method for establishing the diagnosis of mitral stenosis and/or regurgitation, and to some extent, grading the severity of mitral valve disease using pattern recognition. It provides an original approach to the understanding of mitral haemodynamic disturbances on a beat-to-beat basis.

The paramount importance of the knowledge of instantaneous transmitral blood flow for our understanding of/and our ability to diagnose accurately mitral valve disease has been recently stressed (Kalmanson, 1976). We have previously shown the diagnostic use of continuous wave Doppler ultrasonic transseptal catheterisation of the mitral valve for such purposes (Kalmanson et al., 1975a, b). Unfortunately, the cumbersome and traumatic nature of such a procedure somewhat restricts its routine application in clinical cardiology and therefore led us to search for a non-invasive technique for investigating mitral valve flow.

In 1973, Baker (Johnson et al., 1973) devised a new technique for localising the origin of heart murmurs by demonstrating the sites of turbulent

${ }^{1}$ Partly supported by a grant of I.N.S.E.R.M. (C.N.A.M.T.S.).

Received for publication 6 September 1976 blood flow using the combined transcutaneous pulsed Doppler and conventional echocardiographic procedures. At our request, the audio frequency output signal of the pulsed Doppler was demodulated by means of a frequency-to-voltage converter and a single continuous curve was thus substituted for the frequency spectral display previously necessary to interpret the output signal. A new method was devised and tested by our group for recording blood flow velocity patterns from the heart valves. The encouraging results are presented here.

\section{Subjects and methods}

We first studied a control group of 30 subjects, 13 women and $17 \mathrm{men}$, ranging in age from 7 to 40 years, all with normal hearts or in whom organic or 
Table 1 Haemodynamic data of patients with pure mitral stenosis

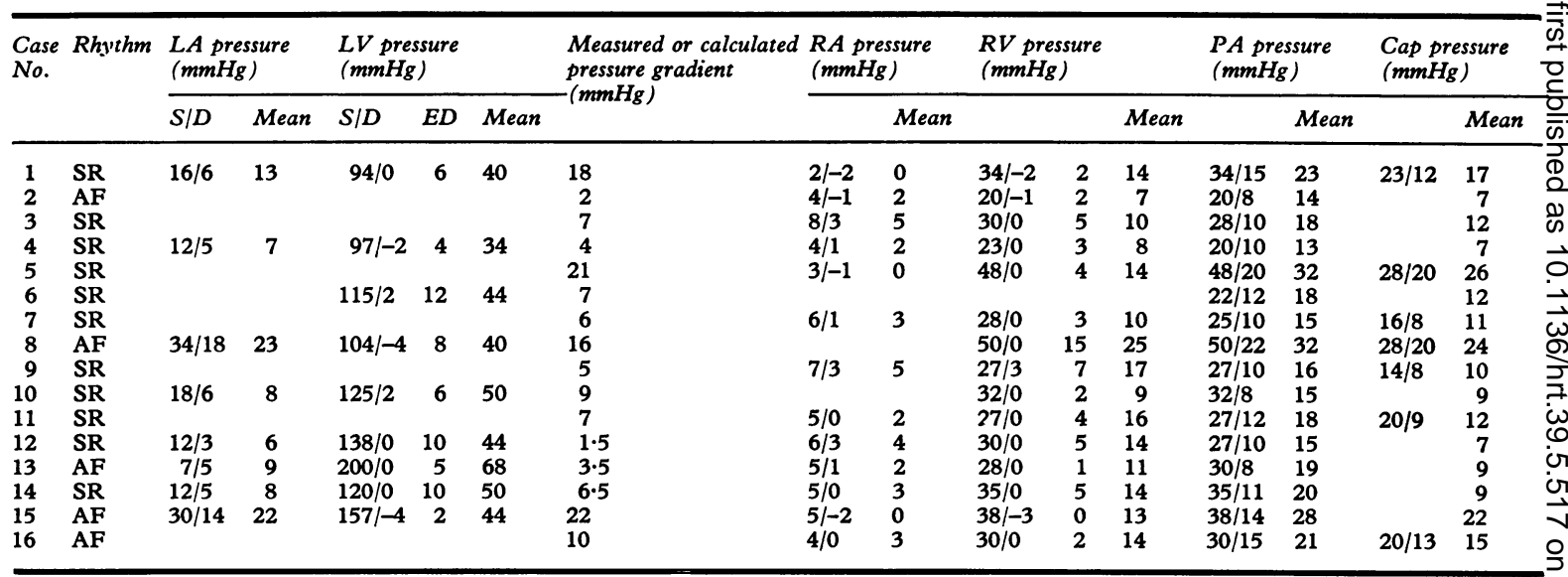

LA, left atrium; S/D, systolic/diastolic; LV, left ventricle; RA, right atrium; PA, pulmonary artery; Cap, capillary; CI, cardiac index; MS, mitral stenosis; AS, aortic stenosis; AR, aortic regurgitation; SH, systemic hypertension; SR, sinus rhythm; AF, atrial fibrillation.

Table 2 Haemodynamic data of patients with pure mitral regurgitation

\begin{tabular}{|c|c|c|c|c|c|c|c|c|c|c|c|c|c|}
\hline \multirow{2}{*}{$\begin{array}{l}\text { Case } \\
\text { No. }\end{array}$} & \multirow{2}{*}{ Rhythm } & \multicolumn{3}{|c|}{$L A$ or cap pressure ( $\mathrm{mm} H \mathrm{~g}$ ) } & \multicolumn{3}{|c|}{$L V$ pressure $(\mathrm{mmHg})$} & \multirow{2}{*}{$\begin{array}{l}C I \\
-\left(1 m^{-1} m^{-2}\right)\end{array}$} & \multirow{2}{*}{$\begin{array}{l}\text { Volume of } \\
\text { regurgitation } \\
(\% \text { of } C O)\end{array}$} & \multirow[t]{2}{*}{ Angiography } & \multirow{2}{*}{$\begin{array}{l}M R \\
\text { grading }\end{array}$} & \multirow{2}{*}{$\begin{array}{l}\text { Associated } \\
\text { lesions }\end{array}$} & \multirow[t]{2}{*}{ Surgery } \\
\hline & & $S / D$ & 'v' wave & Mean & & & Mean & & & & & & \\
\hline $\begin{array}{l}19 \\
20 \\
21 \\
22\end{array}$ & $\begin{array}{l}\text { AF } \\
\text { AF } \\
\text { SR } \\
\text { SR }\end{array}$ & $\begin{array}{l}12 / 8 \\
14 / 12\end{array}$ & $\begin{array}{l}30 \\
36\end{array}$ & $\begin{array}{l}17 \\
20\end{array}$ & $\begin{array}{l}130 / 2 \\
104 / 4 \\
120 / 0\end{array}$ & $\begin{array}{r}10 \\
15 \\
5\end{array}$ & $\begin{array}{l}44 \\
40 \\
38\end{array}$ & $\begin{array}{l}2 \cdot 4 \\
3 \cdot 9 \\
2-3 \\
1\end{array}$ & $\begin{array}{l}15 \\
20 \\
18 \\
/\end{array}$ & $\begin{array}{l}\text { Antr MV } \\
\text { prolapse }\end{array}$ & $\begin{array}{l}2 \\
2 \\
2 \\
1\end{array}$ & $\begin{array}{l}\text { My inf } \\
\text { My inf }\end{array}$ & + \\
\hline $\begin{array}{l}23 \\
24 \\
25\end{array}$ & $\begin{array}{l}\text { SR } \\
\text { AF } \\
\text { SR }\end{array}$ & $\begin{array}{l}19 / 5 \\
12 / 10 \text { cap }\end{array}$ & $\begin{array}{l}52 \\
45\end{array}$ & $\begin{array}{l}20 \\
48\end{array}$ & $\begin{array}{l}100 / 0 \\
105 / 2 \\
140 /-1\end{array}$ & $\begin{array}{r}12 \\
6 \\
10\end{array}$ & $\begin{array}{l}36 \\
30 \\
46\end{array}$ & $\begin{array}{l}1.6 \\
1.5 \\
l\end{array}$ & $\begin{array}{l}25 \\
30 \\
1\end{array}$ & $\begin{array}{l}+ \\
\text { Postr MV } \\
\text { prolapse }\end{array}$ & $\begin{array}{l}3 \\
3 \\
1\end{array}$ & & + \\
\hline
\end{tabular}

For other abbreviations, see Table $1 . \quad M V$, mitral valve; $M y$ inf, myocardial infarct.

Table 3 Haemodynamic data of (catheterised) patients with associated mitral stenosis and regurgitation

\begin{tabular}{|c|c|c|c|c|c|c|c|c|c|c|c|c|c|c|}
\hline \multirow{2}{*}{$\begin{array}{l}\text { Case } \\
\text { No. }\end{array}$} & \multirow{2}{*}{ Rhythm } & \multicolumn{3}{|c|}{$L A$ pressure $(m m H g)$} & \multicolumn{3}{|c|}{$L V$ pressure $(\mathrm{mmHg})$} & \multirow{2}{*}{\multicolumn{2}{|c|}{$\begin{array}{l}\text { LA-LV } \\
\text { pressure } \quad C I \\
(m m H g)\left(1 m^{-1} m^{-2}\right)\end{array}$}} & \multirow{2}{*}{$\begin{array}{l}\text { Volume of } \\
\text { regurgitation } \\
(\% \text { of } C O)\end{array}$} & \multirow{2}{*}{$\begin{array}{l}\text { Calculated } \\
\text { mitral } \\
\text { area }\left(\mathrm{cm}^{2}\right)\end{array}$} & \multirow{2}{*}{$\begin{array}{l}\text { MS } \\
\text { grading }\end{array}$} & \multirow{2}{*}{$\begin{array}{l}M R \\
\text { grading }\end{array}$} & \multirow{2}{*}{$\begin{array}{l}\text { Associated } \\
\text { lesions }\end{array}$} \\
\hline & & $S / D$ & 'v' wave & Mean & S/D & & Mean & & & & & & & \\
\hline
\end{tabular}

For other abbreviations, see Table $1 . \quad$ TR, tricuspid regurgitation; PH, pulmonary hypertension. 


\begin{tabular}{|c|c|c|c|c|}
\hline $\begin{array}{l}S I \\
\left(1 m^{-1} m^{-2}\right)\end{array}$ & $\begin{array}{l}\text { Calculated } \\
\text { mitral area } \\
\left(\mathrm{cm}^{2}\right)\end{array}$ & $\begin{array}{l}\text { MS } \\
\text { grading }\end{array}$ & $\begin{array}{l}\text { Associated } \\
\text { lesions }\end{array}$ & Surgery \\
\hline $\begin{array}{l}3 \\
2.1 \\
3.5 \\
2.7 \\
2.8 \\
3.7 \\
2.6 \\
3.5 \\
2.1 \\
2.8 \\
3.0 \\
2.5 \\
2.0 \\
3.7 \\
2.0 \\
1.88\end{array}$ & $\begin{array}{l}1.2 \\
2.2 \\
2.0 \\
2.3 \\
0.7 \\
2 \\
0.9 \\
1.7 \\
1.6 \\
1.5 \\
1.8 \\
3 \\
2.5 \\
1.7 \\
0.6 \\
1.0\end{array}$ & $\begin{array}{l}3 \\
1 \\
1 \\
1 \\
3 \\
1 \\
3 \\
2 \\
2 \\
2 \\
2 \\
1 \\
1 \\
2 \\
3 \\
3\end{array}$ & $\begin{array}{l}\mathrm{AS}+\mathrm{AR} \\
\mathrm{AR} \\
\mathrm{SH} \\
\mathrm{AR}\end{array}$ & $\begin{array}{l}+ \\
+ \\
+\end{array}$ \\
\hline
\end{tabular}

functional mitral valve disease was excluded by classical investigation procedures.

Thirty-one patients were studied, 21 women and 10 men, ranging in age from 23 to 71 years (Tables 1,2 , and 3). They included 16 patients with pure mitral stenosis, 9 with pure mitral regurgitation, 6 with associated mitral stenosis and regurgitation. The mitral stenosis was all of rheumatic origin. The aetiology of the mitral regurgitation included rheumatic heart disease (3), papillary muscle dysfunction (2), prolapse of the mitral valve (3), floppy valve (1); 6 had associated aortic and/or tricuspid valve lesions; 21 were in sinus rhythm, and 10 in atrial fibrillation. All patients but 2 underwent either a left or a right heart catheterisation, or both using a Telco manometer. ${ }^{1}$ In addition, 10 patients had a continuous wave Doppler transseptal catheterisation in order to determine the ultrasonic mitral valve flow velocity pattern using the directional Sonicaid Doppler (Sonicaid BV 180) cathetertip velocimeter, using a previously described technique (Kalmanson et al., 1975a).

Cardiac output was measured by the dye (indocyanine green) dilution technique using the Waters ${ }^{2}$ apparatus. Mitral valve area was calculated using the Gorlin formula with correction for any mitral regurgitation which was assessed by dye dilution and/or left ventriculography.

\section{CLASSIFICATION OF PATIENTS}

We used 4 grades of severity: (0, no lesion; 1, mild; 2 , moderate; 3 , severe). The classification of

${ }^{1}$ Thomson-Telco, 92212 Saint-Cloud, France.

${ }^{2}$ Waters, Rochester, Minnesota, U.S.A.

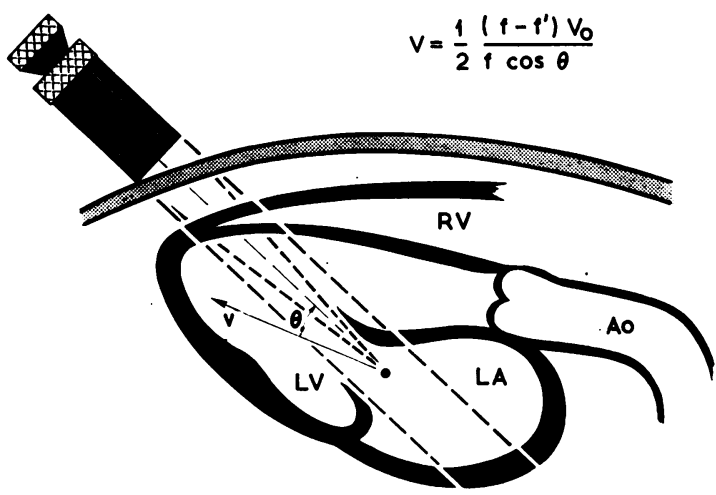

Transcutaneous MV flow velocity recording by pulsed Doppler + echo

Fig. 1 The ultrasonic pulsed beam is backscattered (a) from the cardiac structures and displayed in the $M$-mode on the oscilloscope; (b) from a small sample of blood at the annulus according to the Doppler principle. Orientation of the beam towards a direction parallel to the inflow of blood into the left ventricle $(v)$ is crucial in order to minimise the incidence of the unknown angle $\theta$ and obtain a good 'Doppler' audible signal.

the pure or predominant lesion on this four-point scale was based partly on the severity of the symptoms, and partly on the clinical presentation (bedside examination, $x$-ray, electrocardiogram, and clinical course) as well as on the haemodynamic data. For mitral stenosis, grading was based on the left atrial to left ventricular pressure gradient and particularly on the calculated mitral area: greater than $1.8 \mathrm{~cm}^{2}$ for grade 1 , from 1.8 to $1.3 \mathrm{~cm}^{2}$ for grade 2 , and less than $1.3 \mathrm{~cm}^{2}$ for grade 3 ; it was appreciated that borderline cases might occasionally overlap these categories. For mitral regurgitation grading was based on a large atrial ' $v$ ' wave, dye dilution curves, and/or angiocardiography, and for both lesions, surgical findings, when appropriate. Lesions associated with those of the mitral valve were also classified in 4 grades depending on the clinical and haemodynamic data, and surgical findings. The haemodynamic data are summarised in the Tables.

\section{TECHNIQUE}

The range-gated ultrasonic pulsed Doppler technique has been described elsewhere (Baker, 1970; Peronneau et al., 1970). The principle, the apparatus, and use of Doppler combined with echocardiography using a spectral analyser has also been described (Johnson et al., 1973). Here it is, therefore, only necessary to emphasise the essential characteristics and the modifications caused by the use of an analogue readout (Fig. 1). 


\section{(a) The Doppler principle}

The instantaneous flow velocity of a small $(2 \times 4 \mathrm{~mm})$ tear-drop shaped sample volume of blood is proportional to the frequency shift between ultrasonic beams emitted to, and back-scattered from, the red blood cells of the sample (Fig. 1). A range gating system makes it possible to pick up any desired sample of blood along the axis of the ultrasonic beam within the depth limitations $(5$ to $17 \mathrm{~cm})$.

\section{(b) Apparatus}

We used the ATL ${ }^{1}$ blood flow meter $500 \mathrm{~A}$ with an analogue readout. The small $(0.6 \mathrm{~cm}$ diameter $)$ disc-shaped piezoelectric crystal transmits brief pulses $(1.0$ to $1.5 \mu \mathrm{s})$ of high frequency ultrasound $(3.0 \mathrm{MHz})$, at a pulse repetition rate ranging from 2 to $15 \mathrm{KHz}$. The output wave form is a spectrum of frequencies but can be transformed into a curve by an analogue converter whose readout may be recorded on a strip chart. The use of a zerocrossing detector for obtaining an analogue readout unquestionably underestimates the true value of flow velocity in highly turbulent flow, which is always present in mitral stenosis and most often in mitral regurgitation. It is, therefore, worth ${ }^{1}$ ATL, Bellevue, Washington, U.S.A.

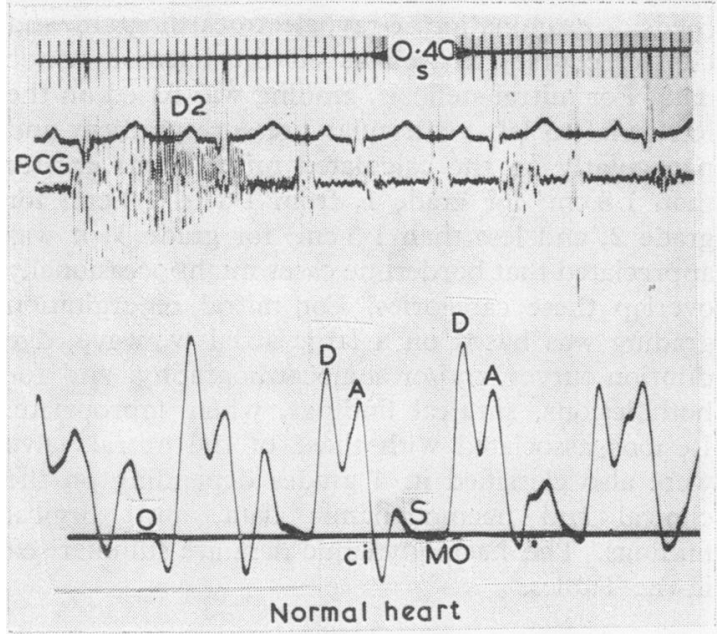

Fig. 2 Normal flow velocity pattern. From top to bottom: Lead II of electrocardiogram, phonocardiogram at the second left interspace, flow velocity trace. Distance between small vertical bars equals : $0.04 \mathrm{~s}$. The systolic curve begins with a brief negative deflection (ci) caused by the isometric contraction of the left ventricle, thereafter the curve rises abruptly, and follows closely the baseline during the remainder of systole. During diastole, the curve rises sharply and presents two successive triangularshaped waves. $D$, initial filling wave; $A$, atrial contraction wave; $M O$, point of true mitral opening.

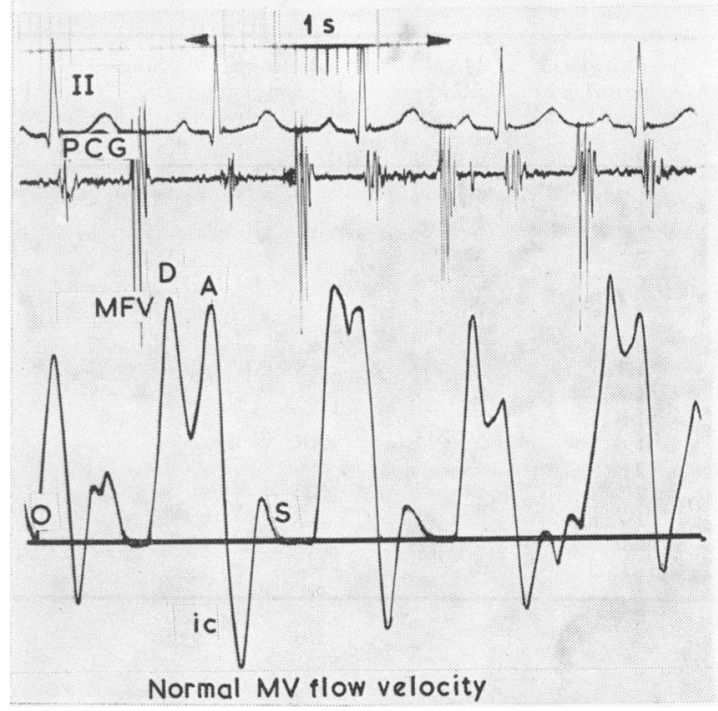

Fig. 3 Normal mitral flow velocity patterns. Depending on the length of the $R R$ interval, the $D$ and $A$ waves may become more or less fused.

emphasising that the method presently proposed relies exclusively on pattern recognition and not on accurate measurements of absolute velocities as will be subsequently shown, but this drawback does not affect in the least the diagnostic value of our method. The Doppler signal can also be fed to a loud speaker. The apparatus includes a conventional

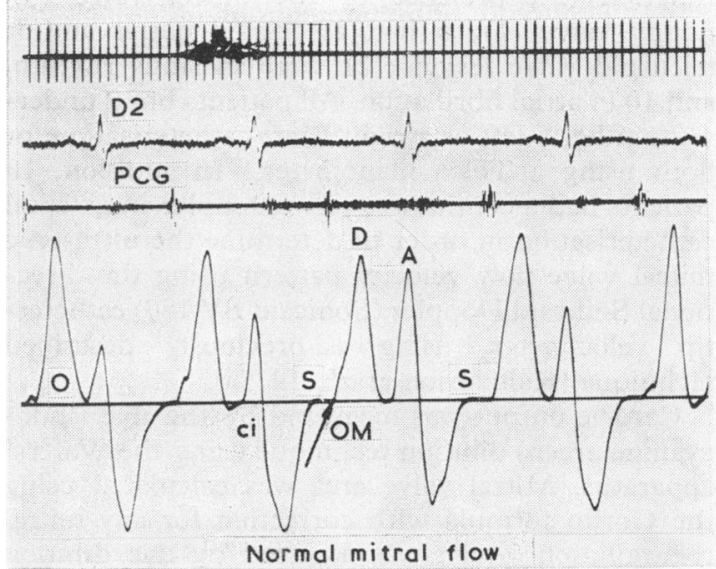

Fig. 4 Normal mitral flow pattern. After the negative deflection at the beginning of systole, the curve became upright, with a small delay probably caused by an artefact. Such an initial negative wave should not be mistaken for a mitral regurgitation wave. Note that in Fig. 2, 3, or 4 there is no intermediate segment elicited between the normal $D$ and $A$ waves. 
echocardiograph with two oscilloscopes (A and $M$ mode). A single transducer operates simultaneously both for the Doppler and the echocardiographic procedures (Fig. 1). The location of the Doppler gate can be displaced using a knob and monitored on the scopes. The approximate time delay determined by comparison with an electromagnetic flowmeter was $65 \mathrm{~ms} \pm 10$ per cent. It may be considered that the device is linear within 5 per cent within the range from 0 to $100 \mathrm{~cm} / \mathrm{s}$ for steady flow.

\section{(c) Recording technique}

In the first place, the transducer coated with conductive jelly is placed on the skin near the fourth left intercostal space of the reclining patient, and is used, according to the conventional echo-technique, to obtain the mitral valve anterior leaflet and the annulus on both scopes. Thereafter, and in contrast to the previously described echo-Doppler technique for localising heart murmurs, the transducer is moved towards the apex, and its direction is modified in order to orientate the ultrasonic beam parallel to the axis of the mitral annulus which turns out to approximate to the anatomical apexbase axis of the heart. The operator must then make the gate coincide with the site of the mitral annulus. The correct orientation is ascertained when a typical Doppler sound is heard, which indicates that the ultrasonic beam is nearly parallel to blood flow. For each patient, it is possible by modifying the location and orientation of the transducer and/or that of the gate to explore several points of the mitral annulus, including the centre, the commissural areas, and the intermediate regions. Failure to explore carefully the mitral annulus may lead to a lesion being overlooked or its severity being underestimated. It is also necessary to take records at various times in the respiratory cycle, and with the breath held.

\section{(d) Recordings}

The traces were recorded in apnoea or very quiet respiration, with the patients lying on their backs or in the left lateral position, and were inscribed on an 8-channel photographic Irex ${ }^{1}$ recorder, simultaneously with lead II of the electrocardiogram and frequency selecting phonocardiogram. Chart speed was $50 \mathrm{~mm} / \mathrm{s}$, unless otherwise specified. The distance between two successive vertical bars equals $40 \mathrm{~ms}$.

\section{Results}

The recorded curves represent the velocity patterns at different points of the mitral annulus. Since the device is directional, a positive or negative sign of velocities depends on the orientation of the transducer with respect to the direction of blood flow. ${ }^{2}$ Irex, Mahwah, New Jersey, U.S.A.

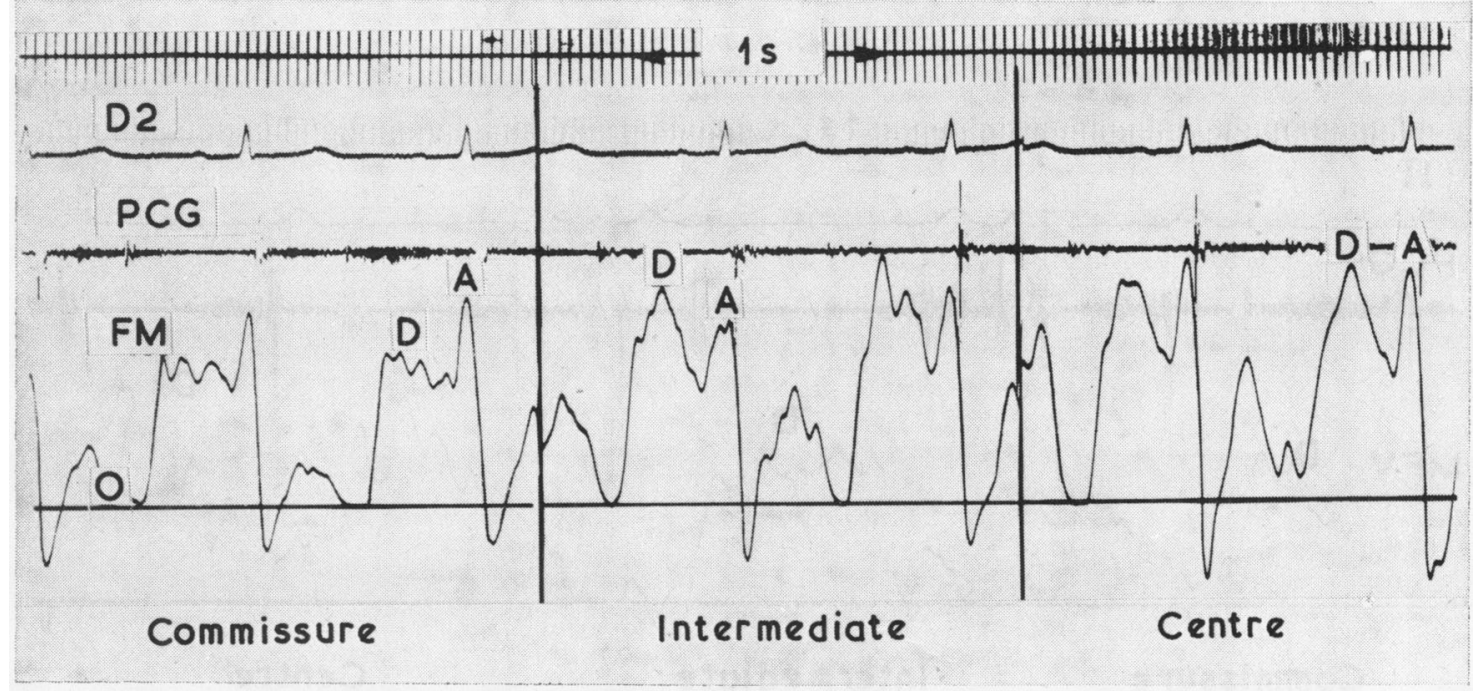

Fig. 5 Mitral flow velocity patterns in pure mild stenosis with sinus rhythm. Right: recording at the centre of the orifice, the $D$ wave is normal, and discloses no indentations. Middle: intermediate region, some small indentations appear either late on the ascending limb of the $D$ wave which retains a normal slope, or at the beginning of its descending limb. Left: recording at the commissure, the summit of the $D$ wave is cut off and replaced by a jagged plateau. Note the absence of any abnormal negative wave throughout the different tracings. Case 12, calculated mitral area: $3 \mathrm{~cm}^{2}$ 
By convention, positive velocities apply to blood flowing from the left atrium into the left ventricle. Negative velocities apply to blood regurgitating from the left ventricle into the left atrium and also to atrial blood pushed back by an upward displacement of a closed and competent mitral valve.

\section{NORMAL PATTERN OF MITRAL VALVE}

FLOW VELOCITY (Fig. 2, 3, and 4)

In normal subjects, the curve starts at the beginning of systole with a large precipitous and brief negative deflection $(0.05$ to $0.08 \mathrm{~s})$, labelled ci, closely related to the first vibrations of the first heart sound. Then the trace rises more or less rapidly, rejoins the zero line, and keeps close to the latter throughout the remainder of systole. It often shows a small angulation related to the aortic component of the second heart sound, followed by a short positive, diaphasic, or negative segment, ended by a clear-cut inflection point (0). Thereafter, the diastolic part of the curve shows two conspicuous, positive (forward flow) waves: the first early and mid-diastolic, labelled $\mathrm{D}$, starting shortly after the aortic component of the second heart sound, triangular in shape, with a peak occurring (depending on the heart rate) from 0.13 to $0.20 \mathrm{~s}$ after the second sound. It is followed by an end-diastolic wave, labelled $\mathrm{A}$, also triangular in shape, starting from 0.10 to $0.12 \mathrm{~s}$, and reaching a peak from 0.16 to $0.20 \mathrm{~s}$ after the onset of the $P$ wave. Peak velocities of the D and A waves vary inversely, de- pending on heart rate and respiration. For normal or slow rates the $D$ wave is larger than the $A$ wave and inversely for faster rates. In cases with long PR intervals (equal to or greater than $0.20 \mathrm{~s}$ ), the descending limb of the $A$ wave falls precipitously and overrides the zero line before the occurrence of the first heart sound.

Other patterns, showing small variations, can be elicited, depending on the site of the recording. The negative initial ci deflection may be absent, or sometimes prolonged for a short period of early systole (Fig. 4). In subjects with a heart rate above $60 / \mathrm{min}$, never more than two filling waves were recorded, whereas in subjects with a slower heart rate, an intermediate segment, usually in the form of a small bulge, occurred between the initial $\mathrm{D}$ and the enddiastolic A waves.

\section{PATIENTS WITH MITRAL VALVE DISEASE} Pure mitral stenosis (16 cases)

In all patients, the diastolic part of the curves consistently showed anomalies of the D wave, of various patterns, depending on the severity of the lesion and on the site of the recording with respect to the centre and the commissural areas.

Mild stenosis (Fig. 5) (6 patients) At the centre of the annulus a normal pattern was consistently recorded, including a smooth $\mathrm{D}$ wave of normal timing and amplitude. When moving from the centre toward the commissures, small indentations

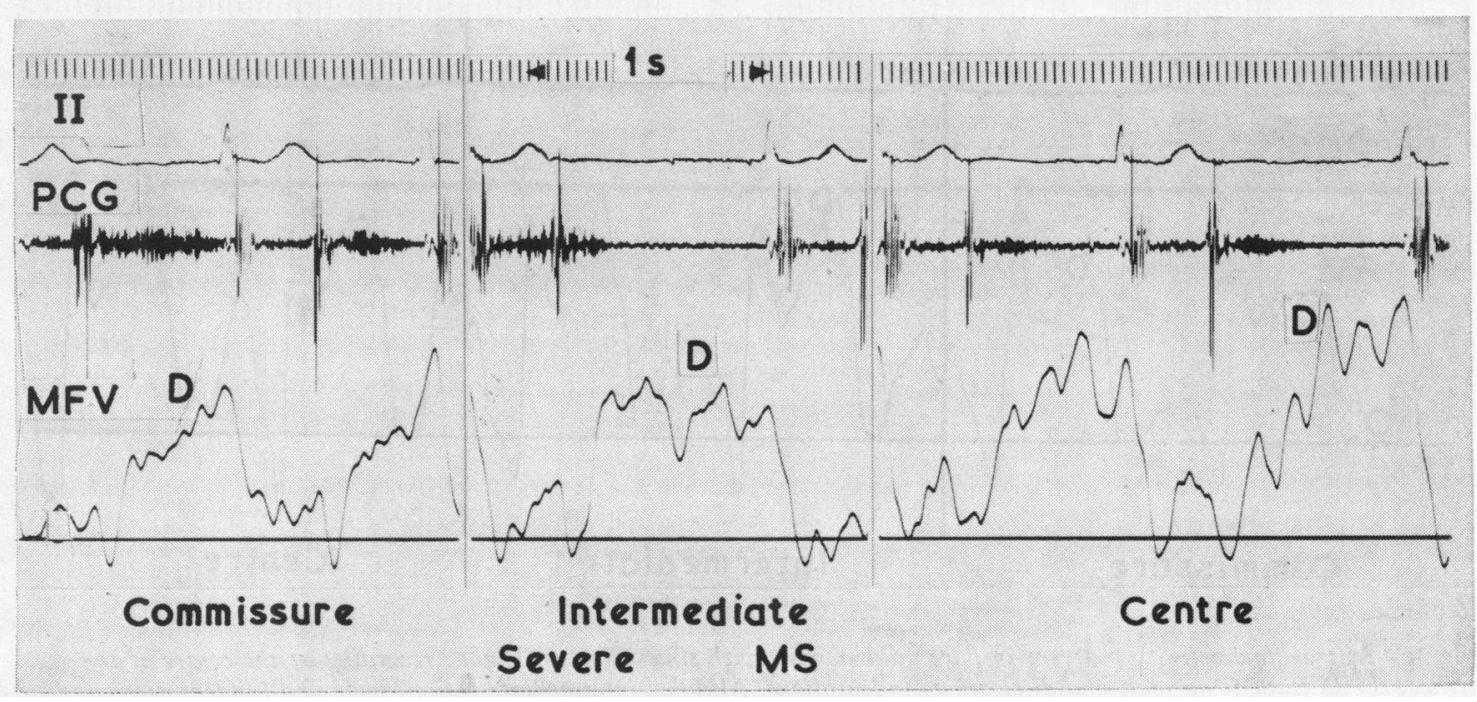

Fig. 6 Mitral flow velocity patterns in severe pure stenosis with atrial fibrillation. At no point can a normal pattern be elicited. All tracings show a cut-off, serrated, either plateau-like or ascending $D$ wave. Case 15, calculated mitral area : $0.6 \mathrm{~cm}^{2}$. 
occurred, late on the ascending limb, at the summit or even on the descending limb of the $\mathrm{D}$ wave. Noticeable anomalies were recorded only at the commissures, in the form of coarse indentations of most of the D wave, whose summit was cut off and lowered. In 3 patients, a conspicuous notch encroached on the ascending limb of the $D$ wave approximately $65 \mathrm{~ms}$ after the occurrence of an opening snap recorded on the phonocardiogram. In the patients with atrial fibrillation, the same modifications were observed, save for the absence of the A wave.

Severe stenosis (Fig. 6 and 7) (5 patients) In all patients the velocity patterns were conspicuously distorted, showing at all sites early and coarse indentations of the $\mathrm{D}$ wave. At the commissural areas, the $\mathrm{D}$ wave was noticeably decreased in amplitude, with an ascending limb having a smaller slope than normal; the peak was either cut off and replaced by a serrated plateau or conspicuously delayed. The A wave was usually higher than the D wave (Fig. 7). At the centre the pattern was equally distorted, but the velocities tended to be larger, with an A wave of increased amplitude.

Moderate stenosis (4 patients) Patterns intermediate between the two previous ones were found consistently. The normal pattern could never be elicited, even at the centre. However, the overall velocities tended to be larger, the indentations occurred later,

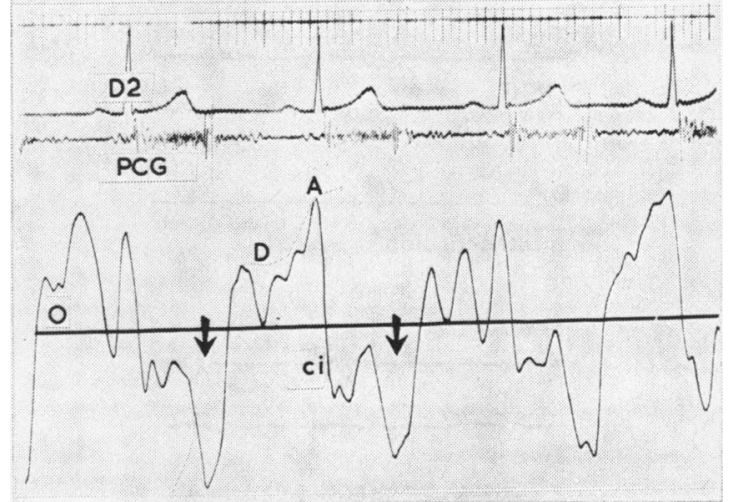

Fig. 8 Flow velocity pattern in mild pure regurgitation. Case 25 with mitral valve prolapse and regurgitation less than 10 per cent of cardiac output. Note the brief and conspicuous reversal flow wave at the end of systole. The diastolic part of the curve is depressed because of the difference in spatial direction of the filling wave and of the regurgitating jet confirmed by angiography.

and were smaller than in patients with severe stenosis.

Pure mitral regurgitation (9 patients)

Mild regurgitation (3 patients) The normal systolic $S$ wave was replaced by a small negative wave, in one case prevailing at end systole (Fig. 8). The diastolic pattern remained normal or was decreased.

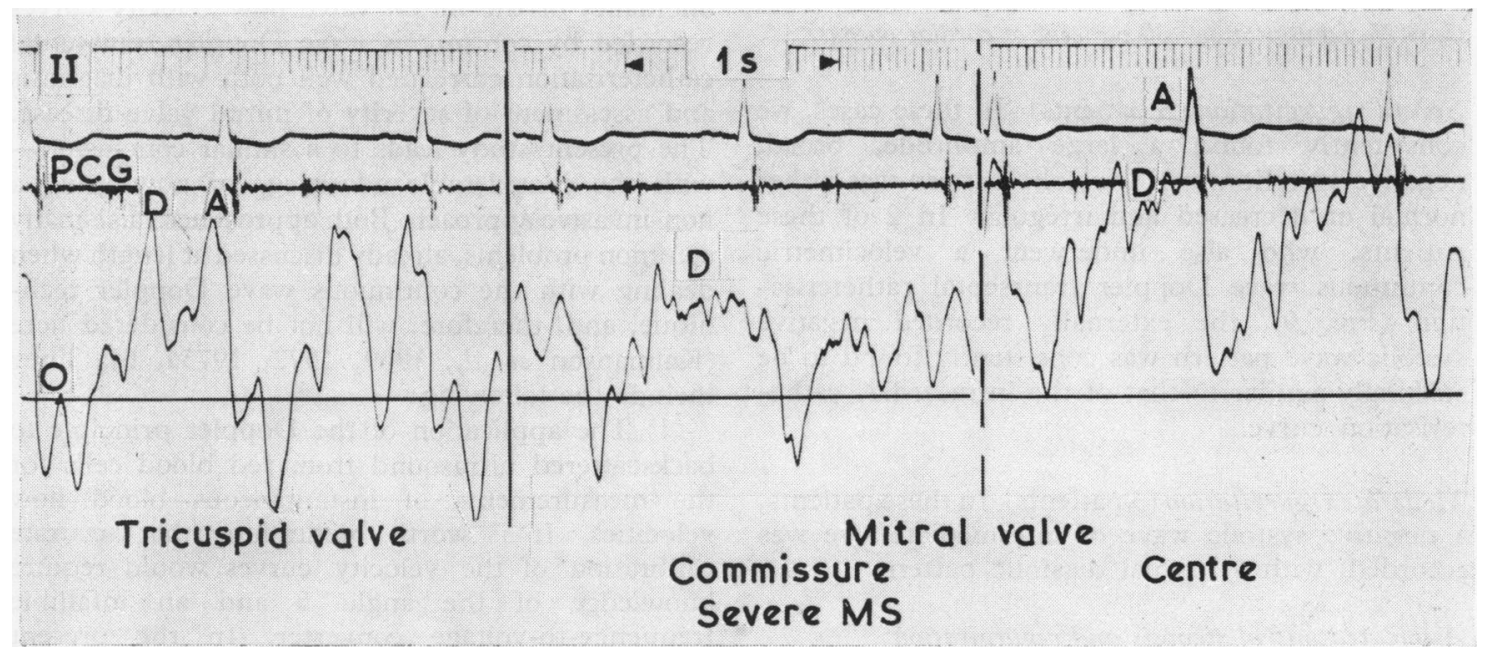

Fig. 7 Mitral flow velocity patterns in severe pure stenosis with sinus rhythm. Compare the normal aspect of the flow velocity recorded at the tricuspid annulus (left) with those obtained at the centre (right) or at the commissures (middle). At the centre, the D wave is replaced by an irregular, sawtooth-like ascending segment, and at the commissure it is cut off and presents a jagged plateau. There is no abnormal negative wave during systole. Case 5, calculated mitral area: $0.7 \mathrm{~cm}^{2}$. 


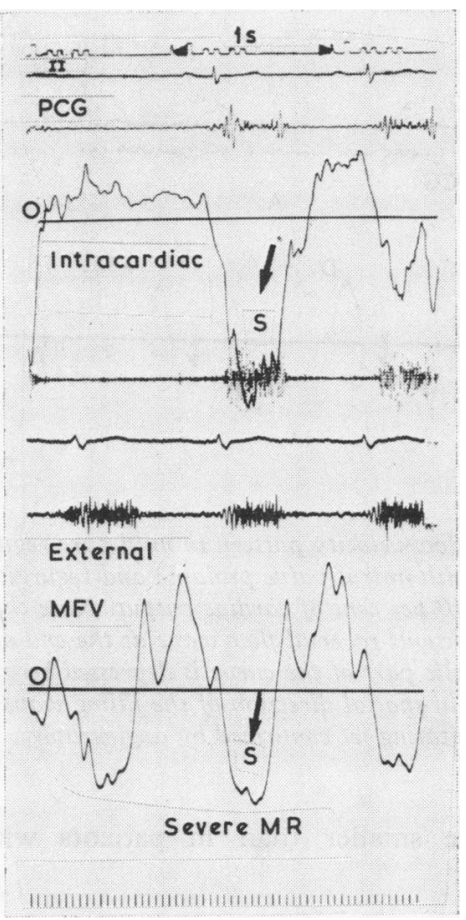

Fig. 9 Flow velocity pattern in case of major regurgitation. Comparison with curve recorded by cardiac catheterisation. Lower row : transcutaneous recording. The systolic wave is replaced by a deep negative wave.

Note the similarity of this systolic pattern with that of the curve recorded in the same patient during Doppler transseptal catheterisation of the mitral valve (upper row). Case 23, regurgitation: 30 per cent of cardiac output.

Severe regurgitation ( 3 patients) In these cases, we consistently found a large amplitude, broad, negative systolic wave. Diastolic pattern was either normal or decreased and irregular. In 2 of these patients, who also underwent a velocimetric continuous wave Doppler transseptal catheterisation (Fig. 9), the externally recorded negative systolic wave pattern was consistently found to be strikingly similar to that of the intracardiac catheterisation curve.

Moderate regurgitation (3 patients) In these patients, a negative systolic wave of intermediate size was recorded, with a normal diastolic pattern.

\section{Associated mitral stenosis and regurgitation (6 patients)}

Mild or moderate stenosis and mild regurgitation (3 patients) A negative systolic $S$ wave of small amplitude at one or several points of the annulus, or of moderate amplitude in the centre, was con- sistently associated with typical anomalies of the D wave found in the patients with mild or moderate stenosis (Fig. 10 and 11).

Severe stenosis and mild to moderate regurgitation (2 patients) Early and coarse irregular indentations of the $\mathrm{D}$ wave were associated with a negative systolic wave of small to moderate amplitude, usually at limited points, most often in the centre (Fig. 12). In 3 patients in whom the cardiac catheterisation did not disclose any regurgitation, we noted an associated small negative systolic either at the centre or at the commissure.

In this group of patients, the velocimetric continuous wave Doppler transseptal catheterisation, whenever available, disclosed a strikingly similar pattern for both diastolic and systolic anomalies (Fig. 12).

Severe mitral stenosis with important regurgitation (1 patient) Early beginning and coarse indentations of the $\mathrm{D}$ wave at all points of the annulus were associated at one or several points with a negative systolic wave of large amplitude (Fig. 13).

In 3 other patients (cases 1,8 , and 14), in whom the conventional procedures did not disclose any regurgitation, the pulsed Doppler showed a minute ( 2 cases) to moderate (1 case) negative systolic wave.

\section{Discussion}

It has been shown previously that particular anomalies of the mitral valve flow velocity curves recorded by continuous wave Doppler transseptal catheterisation correlated well both with diagnosis and assessment of severity of mitral valve disease. The present study leads to a similar conclusionwith the considerable advantage of now using a non-invasive approach. Both approaches raise many common problems, already discussed at length when dealing with the continuous wave Doppler technique, and, therefore, will not be considered here (Kalmanson et al., 1969, 1972, 1975a, b). They include the following:

(1) The application of the Doppler principle to backscattered ultrasound from red blood cells for the measurements of instantaneous blood flow velocities. It is worth mentioning that accurate calibration of the velocity curves would require knowledge of the angle $\theta$ and an infallible frequency-to-voltage converter. In the present state of the art, it is impossible to make quantitative measurements. This shortcoming by no means affects the diagnostic value of the technique which is based on a pattern recognition method.

Incidentally, it is also interesting that the direc- 


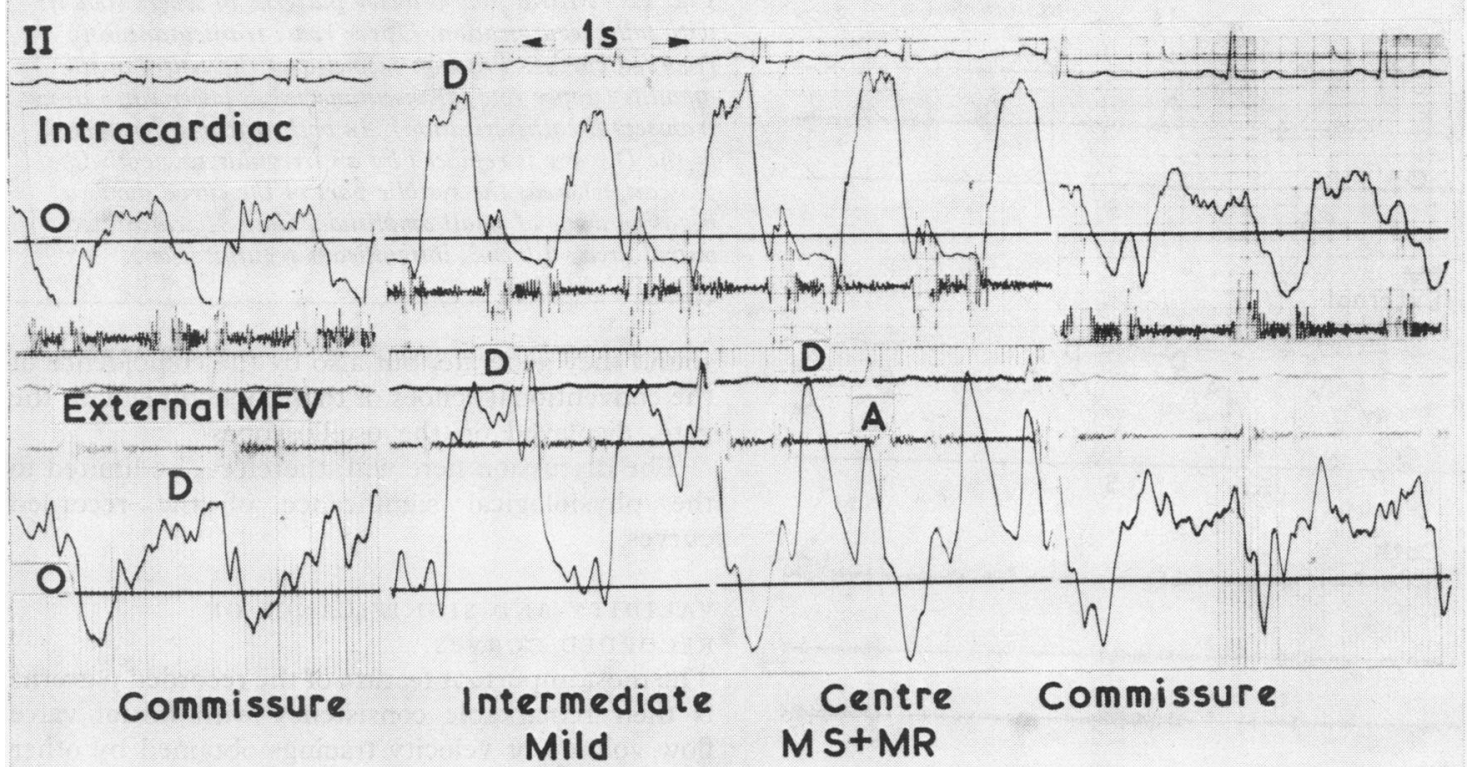

Fig. 10. Mitral flow velocity pattern in moderate stenosis associated with mild regurgitation with sinus rhythm. Comparison with curves recorded in same patient by cardiac Doppler catheterisation (or with intracardiac curves). Upper row : curves recorded by transseptal catheterisation of the mitral annulus. Lower row : transcutaneous recordings. The curves recorded transcutaneously at the centre are normal during diastole and present in particular a normal smooth $D$ wave of normal height. Near the centre, the $D$ wave has still a normal amplitude but discloses late indentations. At the commissures, the $D$ wave is erased and replaced by coarse identations. Note the striking similarity between corresponding curves of both rows, except for the normal pattern which could not be elicited during cardiac catheterisation. At the commissure, there is a small negative systolic wave. Case 14, calculated mitral area: $1 \cdot 7 \mathrm{~cm}^{2}$, insignificant regurgitation.

tion of the left ventricular filling waves, that is that of the main axis of the mitral annulus, tends to parallel the anatomical apex-base axis of the heart (Henry et al., 1959), a situation that minimised the role of the angle $\theta$.

(2) Problems raised by Doppler echoes backscattered from cardiac structures. In the pulsed Doppler technique these echoes can easily be detected, not only by the particular harsh Doppler

Fig. 11 Mitral flow velocity patterns in mild stenosis with minute regurgitation with atrial fibrillation. Upper row : curves recorded by cardiac Doppler catheterisation. Lower row : transcutaneously recorded curves. Left: at the centre in both tracings, the $D$ wave is of normal, large amplitude and smooth, without indentations. Right : at one commissure, both tracings show small indentations. Note the similarity between the intracardiac and transcutaneous patterns. Case 8, mitral calculated area : $1.7 \mathrm{~cm}^{2}$, insignificant regurgitation.

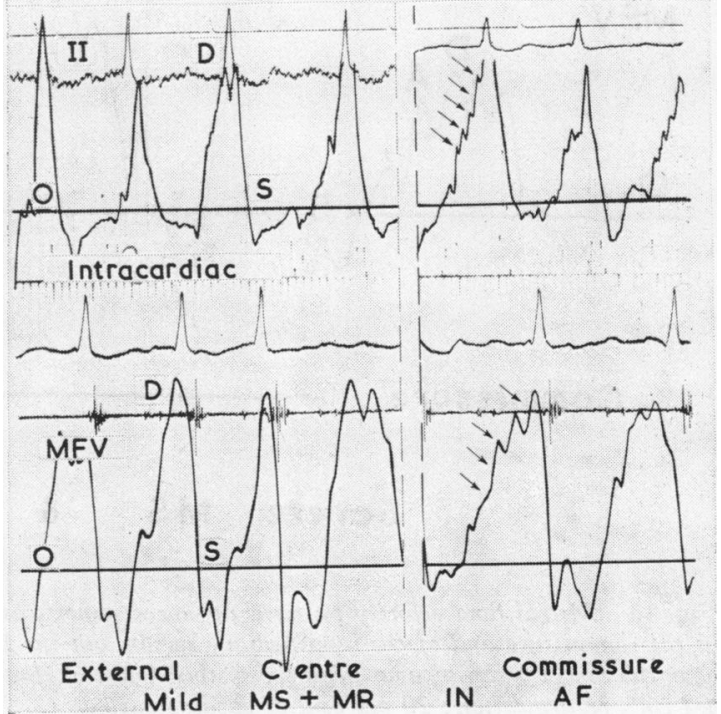




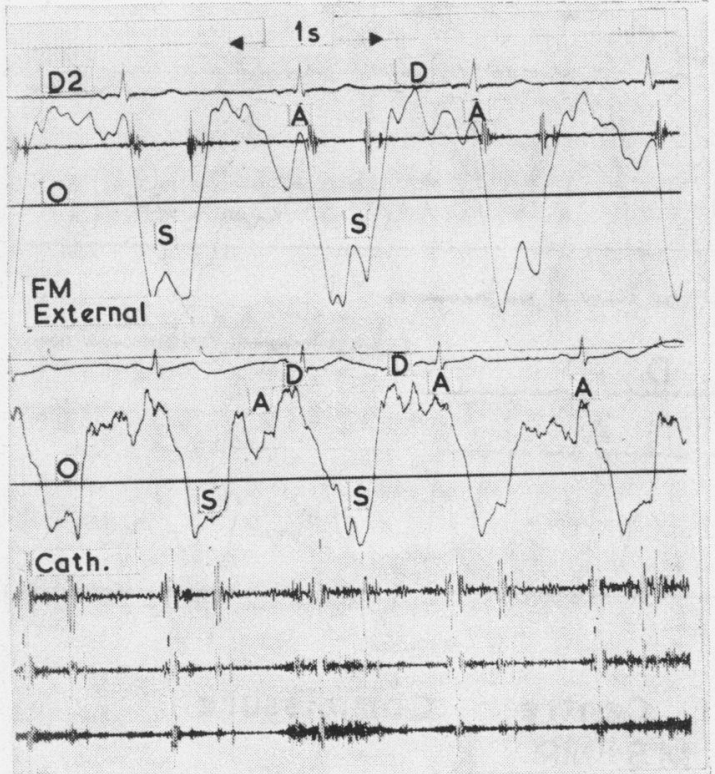

Fig. 12 Mitral flow velocity patterns in severe stenosis with mild regurgitation. Upper row : transcutaneously recorded curves. Tracings recorded at the centre of the annulus (upper row : transcutaneously; lower row : using transseptal catheterisation). In both tracings the summit of the $D$ wave is replaced by an irregular sawtooth-like plateau, whereas the systolic part of the curve shows a negative wave of small amplitude. Case 31, calculated mitral area: $1.0 \mathrm{~cm}^{2}$, insignificant regurgitation.

sound they generate, but also by the coincidence of the conventional echoes of these structures with the gate, displayed on the oscilloscopes.

The discussion here will, therefore, be limited to the physiological significance of the recorded curves.

\section{VALIDITY AND SIGNIFICANCE OF RECORDED CURVES}

The most important feature of the recorded patterns is their remarkable consistency with mitral valve flow volume or velocity tracings obtained by other

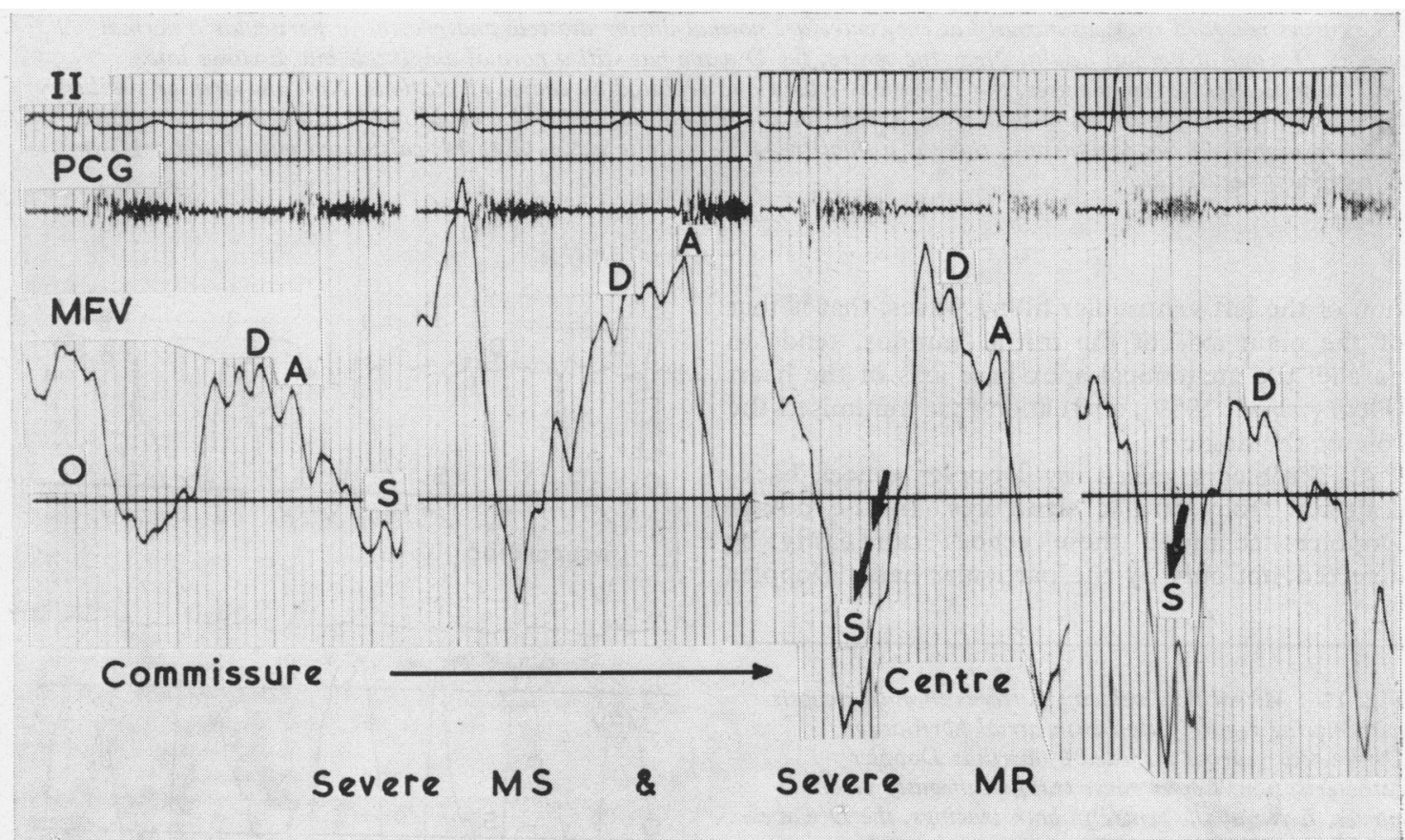

Fig. 13 Mitral flow velocity patterns in severe stenosis associated with an important regurgitation. No backflow at the commissure and at the intermediary region, but the $D$ wave is noticeably cut off and jagged, whereas at and near the centre a conspicuous negative systolic wave of large amplitude is elicited. Case 30, calculated mitral area $1 \cdot 1 \mathrm{~cm}^{2}$, regurgitation: 20 per cent of cardiac output. 
methods either in normal hearts or in animals or patients with mitral valve disease.

(a) The normal pattern recorded by transcutaneous pulsed Doppler ultrasound is strikingly similar to those provided by the electromagnetic cuff flowmeter surgically implanted in dogs or calves (Nolan et al., 1969; Yellin et al., 1970; Folts et al., 1971) and also to those obtained in patients with normal mitral valve using the continuous wave Doppler transseptal catheterisation (Kalmanson et al., 1975a).

(b) The recorded patterns in patients with mitral valve disease are also consistent with those obtained experimentally by Yellin in mitral stenosis and regurgitation (Yellin et al., 1976), and peroperatively by Folts in patients with mitral regurgitation (Folts, 1976). The local velocities shown by the transcutaneous Doppler technique are also similar to the velocity profiles of Whamond (1976) obtained by using the pressure gradient technique in experimental mitral stenosis except perhaps for the amplitude of the wave.

(c) Further convincing evidence was provided in 10 of our patients in whom mitral valve flow velocity patterns could be recorded respectively (though not simultaneously) by intracardiac Doppler catheterisation and by the external technique. Both patterns turned out to be strikingly similar (Fig. 9-12).

It can therefore be concluded that the externally recorded curves actually represent the flow velocity pattern and are endowed with physiological or pathophysiological significance. The conclusions drawn from the studies of the curves recorded at the site of the mitral valve by intracardiac procedures (Kalmanson et al., 1975a, b), also apply to those recorded externally, with the proviso that the peak velocity is not adequately represented whenever turbulent flow is present. In particular, the discussion and comments on the physiological interpretation of the normal curve, its relation to mitral flow involving the variations in size of the mitral annulus, the consequence of a flat profile in normal valves, as well as those in the interpretation of the anomalies shown on the tracings of patients with stenosis or regurgitation hold true for the external flow velocity curves.

It can, therefore, be stated that the indentations occurring on the $\mathrm{D}$ wave are most probably the result of the turbulence generated by the stenosis, whereas the negative systolic wave in mitral regurgitation represents the backflow wave through the mitral annulus.

EFFECT OF VELOCITY PROFILES

(1) The fact that the flow velocity profile in the normal valve is flat indicates that any recording made at any point of the annulus, except for a marginal region less than $5 \mathrm{~mm}$ from the ridge (Taylor and Wade, 1969) is representative of the mitral flow.

(2) This is not true in diseased valves, whose velocity profiles are irregular and asymmetrical (Kalmanson et al., 1975b; Whamond and Taylor, 1976). This stresses the necessity for carefully exploring the various sites of the mitral annulus, lest one type of lesion is overlooked.

\section{DIAGNOSTIC VALUE AND ASSESSMENT} OF SEVERITY

\section{Mitral stenosis}

In our 16 patients with mitral stenosis, we consistently found anomalies of the $D$ wave which consisted of indentations of the ascending limb or of its summit with a decreased slope, at one or several sites of the mitral annulus. These indentations may completely disappear at the centre (mild stenosis), occur late, and be of moderate importance in moderate lesions, and are early and coarse in all points of the mitral annulus in severe stenosis.

\section{Mitral regurgitation}

In our 9 patients with regurgitation, we consistently found a partly or entirely negative systolic wave at one or several sites of the mitral annulus. The amplitude, breadth, and width of the negative wave seem to correlate satisfactorily with the severity of the lesion. However, as previously mentioned, it must be emphasised that finding the negative wave may at times be difficult and time consuming, particularly in cases where the regurgitation is reduced to a localised jet, and careful exploration of the entire annulus at various angles may be necessary to establish the diagnosis.

On the other hand, false positive backflow wave may be present if the recorded blood sample is picked up too far away from the annulus and near the junction between the inflow and outflow tracts, where a physiological negative wave appears (Kalmanson et al., 1972). Therefore, a careful check of the orientation of the transducer as well as of the accurate localisation of the gate at the mitral annulus is a prerequisite for any correct interpretation of a negative systolic wave. It is clear that this qualitative method cannot quantify the regurgitant fraction. However, whenever a careful exploration of the entire annulus is feasible, it seems possible to classify the regurgitation on a threegrade scale.

\section{Combined lesions}

In our 6 patients with associated stenosis and regurgitation, we consistently found the combined 
anomalies described above for each lesion, though these may not show up on the same record, making a thorough investigation of the mitral valve necessary.

Discrepancies In 3 other patients (cases 1, 8, and 14), in whom the conventional procedures did not disclose any regurgitation, the pulsed Doppler showed a minute ( 2 cases) to moderate ( 1 case) negative systolic wave, suggesting that the velocity recording might be more sensitive than the pressure or dye dilution techniques for detecting slight regurgitation.

It therefore can be stated that the transcutaneous pulsed Doppler technique appears to be a reliable aid to the diagnosis and assessment of severity of mitral valve disease, with the above mentioned limitations in respect of mitral regurgitation.

\section{LIMITATIONS OF METHOD}

(1) At present the method is not quantitative, but this does not affect the diagnostic value, since the latter relies exclusively on a pattern recognition method.

(2) The method is not always applicable, particularly in obese patients, or in subjects with dilated thorax or thoracic deformities.

\section{Conclusion}

In spite of some difficulties, the pulsed Doppler technique with analogue readout and associated with echocardiography provides a unique technique for studying mitral blood flow transcutaneously in a simple, safe, and repetitive way. In particular, in spite of some restrictions regarding mitral regurgitation, it turns out to be a reliable method for diagnosing, and assessing the severity of, mitral valve disease, by offering a direct graphic representation of phasic flow velocity disturbances which can be classified using a pattern recognition method. Moreover, it provides a new approach for studying mitral haemodynamic physiology and pathophysiology on a beat-to-beat basis.

The authors thank Dr. Savier who performed the catheterisation and angiography on the patients.

\section{References}

Baker, D. W. (1970). Pulsed ultrasonic Doppler blood flow sensing. I.E.E.E. Transactions on Sonics and Ultrasonics, SU-17, 170-195.

Folts, J. D. (1976). Phasic transmitral blood flow in animals and man obtained without the use of cardiopulmonary bypass. In The Mitral Valve: A Pluridisciplinary Approach. Proceedings of the 1st International Symposium on the Mitral Valve, p. 145. Ed. by D. Kalmanson. E. Arnold, London.

Folts, J. D., Young, W. P., and Rowe, G. G. (1971). Plastic flow through normal and prosthetic mitral valves in unanaesthetized dogs. Fournal of Thoracic and Cardiovascular Surgery, 61, 235-241.

Henry, H., Courbier, R., and Rochu, P. (1959). Les Cavités Cardiaques. Masson, Paris.

Johnson, S. L., Baker, D. W., Lute, R. A., and Dodge, H. T. (1973). Doppler echocardiography. The localization of cardiac murmurs. Circulation, 48, 810-822.

Kalmanson, D. (1976). (Ed.) The Mitral Valve: A Pluridisciplinary Approach. Proceedings of the 1st International Symposium on the Mitral. Valve. E. Arnold, London.

Kalmanson, D., Bernier, A., Veyrat, C., Witchitz, S., Savier, C. H., and Chiche, P. (1975a). Normal pattern and physiological significance of mitral valve flow velocity recorded using transseptal directional Doppler ultrasound catheterization. British Heart Fournal, 37, 249-256.

Kalmanson, D., Toutain, G., Novikoff, N., and Derai, C. (1972). Retrograde catheterization of left heart cavities in dogs by means of an orientable directional Doppler cathetertip flowmeter: a preliminary report. Cardiovascular $R e$ search, 6, 309-318.

Kalmanson, D., Toutain, G., Novikoff, N., Derai, C., Chiche, P., and Cabrol, C. (1969). Le cathétérisme vélocimétrique du coeur et des gros vaisseaux par sonde ultrasonique directionnelle à effet Doppler. Rapport préliminaire. Annales de Médecine Interne, 120, 685-700.

Kalmanson, D., Veyrat, C., Bernier, A., Savier, C. H., Chiche, P., and Witchitz, S. (1975b). Diagnosis and evaluation of mitral valve disease using transseptal Doppler ultrasound catheterisation. British Heart fournal, 37, 257-271.

Nolan, S. T., Dixon, S., Fisher, R., and Morrow, A. (1969). The influence of atrial contraction and mitral valve mechanics on ventricular filling. American Heart fournal, 77, 784-791.

Peronneau, P., Léger, F., Hinglais, J., and Pellet, M. (1970). Vélocimètre sanguin par effet Doppler à émission ultrasonore pulsée. Onde Electrique, 50, 369-389.

Taylor, D. E. M., and Wade, J. D. (1969). Flow through the mitral valve during diastolic filling of the left ventricle. fournal of Physiology, 200, 73P-74P.

Whamond, J. S., and Taylor, D. E. M. (1976). Fluid dynamics of the diseased mitral valve. In The Mitral Valve: A Pluridisciplinary Approach. Proceedings of the 1st Intertional Symposium on the Mitral Valve, p. 187. Ed. by D. Kalmanson. E. Arnold, London.

Yellin, E. L., Laniado, S., Peskin, C., and Frater, R. (1976). Flow studies of experimental mitral stenosis and regurgitation. In The Mitral Valve: A Pluridisciplinary Approach. Proceedings of the 1st International Symposium on the Mitral Valve, p. 195. Ed. by D. Kalmanson. E. Arnold, London.

Yellin, E. L., Silverstein, M., Frater, R., and Peskin, C. (1970). Pulsatile flow dynamics across the natural and prosthetic mitral valve. In Proceedings, Annual Conference on Engineering in Medicine and Biology, November 15-19. Wellesley Press, Wellesley, Massachusetts.

Requests for reprints to Dr. D. Kalmanson, Service de Cardiologie, Fondation A. de Rothschild, 25-29 rue Manin, 75019 Paris, France. 